\title{
Momentum-resolved Raman spectroscopy of bound molecules in ultracold Fermi gas
}

\author{
Zhengkun Fu, Pengjun Wang, Lianghui Huang, Zengming Meng, Jing Zhang ${ }^{\dagger}$ \\ State Key Laboratory of Quantum Optics and Quantum Optics Devices, \\ Institute of Opto-Electronics, Shanxi University, Taiyuan 030006, P.R.China
}

\begin{abstract}
The binding energy of Feshbach molecules from a two component Fermi gas of ${ }^{40} \mathrm{~K}$ atoms has been experimentally measured with the momentum-resolved Raman spectroscopy. Comparing with the radio-frequency spectroscopy, in the present experiment the signal of unpaired (free atoms) and the bound molecules can be directly observed and the binding energy can be simultaneously determined in a single running experiment. The energy-momentum dispersion spectra of the strongly interacting ultracold Fermi gas in BEC side are also measured and reconstructed. The present experimental technology of the momentum-resolved Raman spectroscopy can be easily extended to perform spatially momentum-resolved Raman spectroscopy and to obtain the response spectra of a homogeneous system in the local density approximation.
\end{abstract}

Interacting Fermi gas [1], is a simple, clean and easy controllable system with rich physical behaviors, which provides a new platform for studying the frustrating problems in the condensed matter physics and the quantum simulation of many-body system [2], such as high-temperature superfluidity and BEC-BCS crossover. Magnetic field induced Feshbach resonances present a means to precisely control the interaction of ultracold Fermi atoms in different spin states over several orders of magnitude $3-6]$. This method allows us to create molecules [7, 8] and fermionic superfluidity in balanced and unbalanced components [9 11]. Radio-frequency (RF) spectroscopy has become a powerful tool for studying single-particle excitations in degenerate Fermi gases, such as to probe the pair size [12], energy excitation spectrum [13 16], and paring gap [17 19]. An other important tool in this field is Bragg spectroscopy, which has been used for probing density-density correlations [20] and measuring the universal contact of an interacting Fermi gas [21]. There are also many other detection methods of Fermi pairing proposed theoretically such as Stokes-scattering method [22], interferometric method 23], electromagnetically induced transparency method 24].

The Raman spectroscopy technology [25] has been proposed to probe the one-particle excitation in degenerate Fermi gases. In the Raman process, the atoms in initial state absorb a photon from a Raman laser beam and immediately emit a photon into another Raman laser beam. During this process the atoms are transferred into an other internal state with different momentum. Compared to the RF spectrum, the Raman spectroscopy offers several advantages, e.g., spatial selectivity, tunability of transferred momentum and weak sensitivity to final state interaction [26]. Recently, we have experimentally measured the dispersion of a non-interacting degenerate Fermi gas by means of the momentum-resolved Raman spectroscopy technology [27].

In this letter, we present the first experimental achievement on exploiting Raman spectroscopy to probe Feshbach molecules in ultracold Fermi gas. The Feshbach (a)

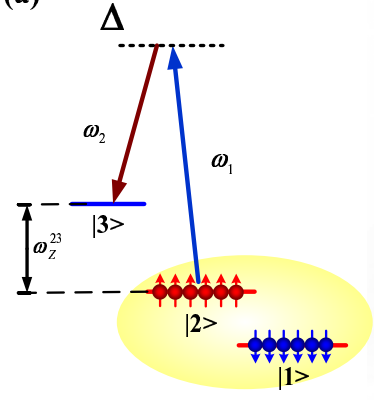

(b)

FIG. 1: (Color online). Geometry and energy level diagram for Raman spectroscopy. a, The atomic level diagram for Raman transition from the state $|2\rangle$ to $|3\rangle$ and the bound molecules formed by the atoms in states $|1\rangle$ and $|2\rangle$. b, Schematic of the Raman spectroscopy experiment. Two Raman beams counterpropagating along $\pm \hat{x}$ illuminate the ultracold Fermi gas and the black arrow indicates the direction of the bias magnetic field $B$.

molecules are created in ${ }^{40} \mathrm{~K}$ atomic gas consisting of an equal mixture of atoms in the $\left|F=9 / 2, m_{F}=-9 / 2\right\rangle$ and $\left|F=9 / 2, m_{F}=-7 / 2\right\rangle$ states by ramping the magnetic field from above the s-wave Feshbach resonance $202.2 \mathrm{G}$ to a value below the resonance. We apply a Gaussian shape pulse of Raman laser to transfer atoms from the state $\left|F=9 / 2, m_{F}=-7 / 2\right\rangle$ to the final state $\left|F=9 / 2, m_{F}=-5 / 2\right\rangle$ and record the momentum distribution of the atoms in $\left|F=9 / 2, m_{F}=-5 / 2\right\rangle$ state by time-of-flight (TOF) absorption image. We observe simultaneously the composition of gas, including unpaired atoms and bound molecules in BEC side and determine the binding energy by the momentum distribution of the atoms in $\left|F=9 / 2, m_{F}=-5 / 2\right\rangle$ state in a single running experiment. The momentum distributions of the atoms in $\left|F=9 / 2, m_{F}=-5 / 2\right\rangle$ state are recorded as a function of the frequency difference between the two Raman lasers, and then the spectral function of the ultracold Fermi gas is reconstructed.

We consider the atomic system with three internal 
states labeled by $|1\rangle,|2\rangle$ and $|3\rangle$, as shown in Fig. 1 (a). There is a interaction between the atoms in state $|1\rangle$ and $|2\rangle$, which forms the molecules with the binding energy $E_{b}$, and the state $|3\rangle$ is non-interacting with both states $|2\rangle$ and $|1\rangle$. The Raman laser frequency-difference is chosen to transfer the atoms in state $|2\rangle$ to $|3\rangle$, but which is not in resonance with any transition. For the Raman process, we define the effective Raman coupling as $\Omega=\Omega_{1} \Omega_{2} / \Delta$, here $\Omega_{i}$ is the Rabi frequency of laser beam $i$ with frequency $\omega_{i}$ and wave vector $\mathbf{k}_{i}, \Delta$ is the detuning between Raman laser field and intermediated excited states. According to energy and momentum conservation, one obtains the momentum transfer $\mathbf{q}_{r}=\mathbf{k}_{1}-\mathbf{k}_{2}$ and the energy shift $\hbar \Delta \omega=\hbar\left(\omega_{1}-\omega_{2}\right)$ of the atoms and it can also be described by expressions of the outgoing channel

$$
\hbar \triangle \omega=E_{Z}^{32}(B)-\epsilon_{|2\rangle}^{\text {Initial }}(\mathbf{k})+\epsilon_{|3\rangle}^{\text {Final }}\left(\mathbf{k}+\mathbf{q}_{r}\right) .
$$

Here $E_{Z}^{32}(B)$ is the Zeeman energy split between the state $|3\rangle$ and $|2\rangle$ in a magnetic field $\mathbf{B} . \epsilon_{|3\rangle}^{\text {Final }}\left(\mathbf{k}+\mathbf{q}_{r}\right)$ is the energy momentum dispersion of atoms in final state $|3\rangle$. The spin flipped atoms have only very weak interactions with the other atoms which means that the dispersion can be expressed with the usual free-particle dispersion: $\epsilon_{|3\rangle}^{\text {Final }}\left(\mathbf{k}+\mathbf{q}_{r}\right)=\hbar^{2}\left(\left|\mathbf{k}+\mathbf{q}_{r}\right|\right)^{2} / 2 m$, where $m$ is the atomic mass. $\epsilon_{|2\rangle}^{\text {Initial }}(\mathbf{k})$ stands for the dispersion of atoms in state $|2\rangle$ strongly interacting with the state $|1\rangle$, which can be reconstructed by the momentum distributions of the atoms in $|3\rangle$ state as a function of the frequency difference of the two Raman lasers,

$$
\epsilon_{|2\rangle}^{\text {Initial }}(\mathbf{k})=E_{Z}^{32}(B)-\hbar \Delta \omega+\hbar^{2}\left(\left|\mathbf{k}+\mathbf{q}_{r}\right|\right)^{2} / 2 m .
$$

The apparatus and the basic experimental methods have been described in our previous publications [28 32], in which the Bose-Fermi mixtures are cooled in magnetic field and transported into an optical trap. The experiment begin with a degenerate Fermi gas about $2 \times 10^{6}$ ${ }^{40} \mathrm{~K}$ in the $\left|F=9 / 2, m_{F}=9 / 2\right\rangle$ internal state, which has been evaporatively cooled to $T / T_{F} \approx 0.3$ with bosonic ${ }^{87} \mathrm{Rb}$ atoms inside the crossed optical trap, where $T$ is the temperature, $T_{F}$ is the Fermi temperature defined by $T_{F}=E_{F} / k_{B}=\frac{(\hbar \bar{\omega})}{k_{B}}(6 N)^{1 / 3}$, and $\bar{\omega}$ is the geometric mean trapping frequency, $N$ is the number of fermions. A $780 \mathrm{~nm}$ laser pulse of $0.03 \mathrm{~ms}$ is used to remove the ${ }^{87} \mathrm{Rb}$ atoms in the mixture without heating of ${ }^{40} \mathrm{~K}$ atoms. Subsequently, the fermionic atoms are transferred into the lowest state $\left|F=9 / 2, m_{F}=-9 / 2\right\rangle$ via a rapid adiabatic passage induced by a radio frequency field of $80 \mathrm{~ms}$ at $4 G$. In order to prepare the Fermi gas ${ }^{40} \mathrm{~K}$ in an equal mixture of $\left|F=9 / 2, m_{F}=-9 / 2\right\rangle$ (regarded as $\left.|1\rangle\right)$ and $\left|F=9 / 2, m_{F}=-7 / 2\right\rangle$ (regarded as $|2\rangle$ ) states, a homogeneous bias magnetic field produced by the quadrupole coils (operating in the Helmholtz configuration) is raised to about $B \approx 219.4 G$ in the $\hat{y}$ direction and then a radio frequency ramp around $47.45 \mathrm{MHz}$ is applied for $50 \mathrm{~ms}$.
It is crucial in the experiment to control the high magnetic field precisely and reduce the drift and AC field noise. The current through the coils is controlled by the external regulator relying on a precision current transducer (Danfysik ultastable 867-60I). An analog current signal is generated from the current transducer which is proportional to the primary current. The current signal is transformed into the voltage signal by a precision resistor with a low temperature coefficient of $15 \mathrm{ppm} /{ }^{\circ} \mathrm{C}$. Then a regulator compares this current value with a given voltage value from a computer. The output error signal from the regulator actively stabilizes the current with the PID (proportional-integral-derivative) controller acting on the MOSFET (metal-oxide-semiconductor fieldeffect transistor). In order to reduce the current noise and decouple the control circuit from the main current, a conventional battery is used to power the circuit.

In order to create molecules, the homogeneous bias magnetic field is ramped from $204 G$ to a value B below the Feshbach resonance located at $202.2 G$ at a rate of about $0.08 \mathrm{G} / \mathrm{ms}$. This procedure would therefore result in a mixture of weakly bound molecules and unpaired atoms in $\left|F=9 / 2, m_{F}=-9 / 2\right\rangle$ and $\mid F=9 / 2, m_{F}=$ $-7 / 2\rangle$ states. To probe the binding energy of molecules by Raman spectroscopy technology, a pair of $773 \mathrm{~nm}$ Raman laser from a Ti-sapphire laser with the frequency difference $\Delta \omega$, counterpropagating along the $\hat{x}$ axis, which couple the two hyperfine states $\left|F=9 / 2, m_{F}=-7 / 2\right\rangle$ $(|2\rangle)$ and $\left|F=9 / 2, m_{F}=-5 / 2\right\rangle(|3\rangle)$ as shown in Fig. $1(\mathrm{~b})$. The momentum transferred to atoms during the Raman process is $\left|\mathbf{q}_{r}\right|=2 k_{r} \sin (\theta / 2)$, where $k_{r}=2 \pi / \lambda$ is the single-photon recoil momentum, $\lambda$ is the wavelength of the Raman beam, and $\theta=180^{\circ}$ is the intersecting angle of two Raman beams. Here, $\hbar k_{r}$ and $E_{r}=\left(\hbar k_{r}\right)^{2} / 2 m=h \times 8.34 k H z$ are the defined units of momentum and energy for the later figures. Two Raman beams are frequency-shifted $-75 \mathrm{MHz}$ and $-122 \mathrm{MHz}$ by two single-pass acousto-optic modulators (AOM) respectively. In this way the relative frequency difference between the two laser beams is precisely controlled by the two signal generators used for two AOMs. After out of the optical fibers, the two Raman beams with intensity $I=50 \mathrm{~mW}$, are intersecting in the atomic cloud with $1 / e^{2}$ radii of $200 \mu m$ and are linearly polarized along $\hat{z}$ and $\hat{y}$ axis directions respectively, which correspond to $\pi$ and $\sigma$ of the quantization axis $\hat{y}$. A Gaussian shape pulse of the Raman laser with a duration time about $70 \mu \mathrm{s}$ is applied to transfer atoms from the initial state $|9 / 2,-7 / 2\rangle$ to the final state $|9 / 2,-5 / 2\rangle$. The pulse is generated by the voltage-controlled $\mathrm{RF}$ attenuators of AOMs' driver. The Gaussian envelop hence results in the elimination of the side lobes in Raman spectra. Here, when the atoms are in Raman resonance (at about $47.2 \mathrm{MHz}$ ) between $\left|F=9 / 2, m_{F}=-7 / 2\right\rangle$ and $\left|F=9 / 2, m_{F}=-5 / 2\right\rangle$ near Feshbach resonance $(202.2$ $G)$, the energy split between $\left|F=9 / 2, m_{F}=-9 / 2\right\rangle$ and 

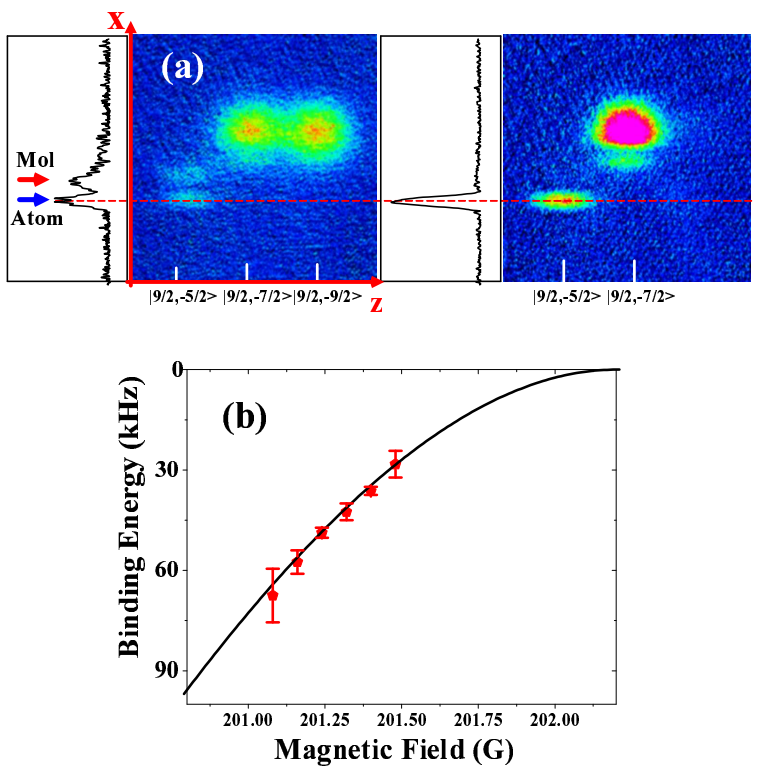

FIG. 2: (Color online). Determining the binding energy of s-wave molecules. a, Absorption images of Fermi gas and integrated optical density of atoms in $\mid F=9 / 2, m_{F}=$ $-5 / 2\rangle$ state along $\hat{z}$ with the mixture and the single spin state $\left|F=9 / 2, m_{F}=-7 / 2\right\rangle$. The red dashed line indicates the same position in $\hat{x}$ for free atoms. Mol: molecules. $\mathbf{b}$, The measured binding energies of molecules on the BEC side of the s-wave Feshbach resonance are plotted versus magnetic fields (red pentagon). The solid line is the theoretical calculation of the binding energy of molecules for ${ }^{40} K$ atoms in $\mid F=$ $\left.9 / 2, m_{F}=-9 / 2\right\rangle$ and $\left|F=9 / 2, m_{F}=-7 / 2\right\rangle$ states.

$\left|F=9 / 2, m_{F}=-7 / 2\right\rangle$ is about $44.8 \mathrm{MHz}$, which is a very large detuning for Raman transition. After the Raman laser pulse, we abruptly turn off the optical trap and the magnetic field, and let the atoms ballistically expand for $12 \mathrm{~ms}$ in a magnetic field gradient applied along $\hat{z}$ and take TOF absorption image along $\hat{y}$. The atoms in different hyperfine states and momentum states are spatially separated and thus can be easily analyzed.

We first measure the function of the binding energy of molecules $E_{b}(B)$ versus the magnetic field on the BEC side of Feshbach resonance. By selecting the appropriate frequency difference $\Delta \omega$ of the two Raman beams, the two distinctly different momentum distributions of the atoms in $|9 / 2,-5 / 2\rangle$ state along $\hat{x}$ appear in a TOF absorption image as shown in Fig. 2(a). The phenomenon is induced by the free atoms and bond molecules with the different momentums, which satisfies Eq. 1 simultaneously when fixing frequency difference $\Delta \omega$ of two Raman beams. In order to recognize the different momentum distributions resulting from the free atoms or the bond molecules, we perform the same procedures and the only difference is to prepare atoms in the single spin state $\left|F=9 / 2, m_{F}=-7 / 2\right\rangle$ instead of the equal mixture. The TOF image only shows a single momentum distribution of the atoms in $|9 / 2,-5 / 2\rangle$ state, which corresponds to the free atoms as shown in Fig. 2(a). The peak corresponding to unpair atoms is narrower and exhibits symmetric shape. The asymmetric peak corresponds to the dissociation of the bound molecules. This result shows that the composition of gas includes the unpaired atoms and bound molecules in BEC side. To determine the binding energy of molecules exactly, we first determine the maximum molecule signal and then fit this curve to extract the distance $\mathrm{x}$ of two peaks of the atoms in $|9 / 2,-5 / 2\rangle$ state from the atom optical density integration along $\hat{z}$ direction. The relationship between the binding energy of molecules and the distance $\mathrm{x}$ of two peaks can be determined by preparing atoms in the single spin state $\left|F=9 / 2, m_{F}=-7 / 2\right\rangle$ and performing respectively two measurements under two different frequency differences between two Raman beams, $\Delta \omega_{1}$ and $\Delta \omega_{2}$. The distance between the atomic momentum distribution peaks measured at the two cases' of $\Delta \omega_{1}$ and $\Delta \omega_{2}$ corresponds to the energy $\hbar\left(\Delta \omega_{1}-\Delta \omega_{2}\right)$. The function of the molecule binding energy versus the magneticfield intensity is plotted in Fig. 2(b). Due to the limit of the momentum distribution of the unpaired atoms and bound molecules, the binding energy of molecules can be directly measured in a single running experiment if the binding energy of molecules is smaller than about 70 $k H z$. When the binding energy of molecules is larger than about $70 \mathrm{kHz}$, we must perform two measurements to determine the positions of molecules and unpair atoms respectively. We compare the measured binding energies near the Feshbach resonance with the black line based on a theoretical calculation of a approximate analytic formula with the requirement of $a \gg r_{0}[7]$

$$
E_{b}=\frac{\hbar^{2}}{m\left(a-r_{0}\right)^{2}}
$$

where $a$ is the scattering length describing the interaction of atoms in states $\left|F=9 / 2, m_{F}=-7 / 2\right\rangle$ and $\mid F=$ $\left.9 / 2, m_{F}=-9 / 2\right\rangle$, and $r_{0} \approx 60 a_{0}$ is the range of Van der Waals potential [7]. The experimental results are consistent with the expected behavior under changing the magnetic field.

Now we reconstruct the spectral function of the Fermi gas on the BEC side by the momentum-resolved Raman spectroscopy. By fixing the magnetic field and changing the frequency difference between the two Raman lasers with a step of $2.5 \mathrm{kHz}$, we obtain the TOF absorption images as function of the frequency difference of Raman lasers as shown in Fig. 3(a). Then we integrate atomic optical density of the atoms in $|9 / 2,-5 / 2\rangle$ state along $\hat{z}$ direction for each image and obtain the momentum distributions in $\hat{x}$. All momentum distributions in $\hat{x}$ of the spin state $|9 / 2,-5 / 2\rangle$ for different frequency differences of the Raman lasers are plotted into the $\left(\Delta \omega, k_{x}\right)$ plane, as shown in Fig. 3(b). According to Eq. 2 and the quadratic energy-momentum dispersion of the final state, the energy-momentum dispersion of the ini- 


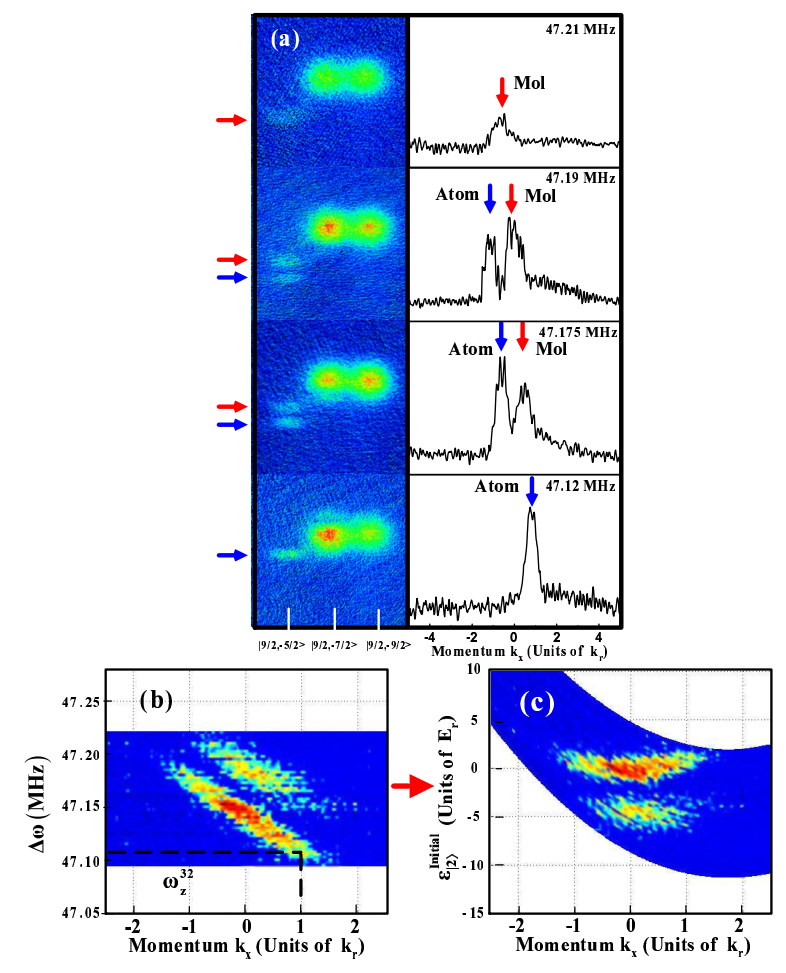

FIG. 3: (Color online). TOF images and the energymomentum dispersion reconstructed by momentumresolved Raman spectroscopy for ultracold Fermi gas. a, Absorption images of Fermi mixture and integrated optical density of atoms in $\left|F=9 / 2, m_{F}=-5 / 2\right\rangle$ state at 201.5 $G$ with Raman frequency difference $\Delta \omega$ from $47.1 \mathrm{MHz}$ to 47.22 MHz. b, The plot is intensity map of the atoms in $\left|F=9 / 2, m_{F}=-5 / 2\right\rangle$ state in the $\left(\Delta \omega, k_{x}\right)$ plane. The atomic density is displayed with the pseudocolor. The blue shade regions correspond to the atomic density of zero. c, The translated intensity spectrum shows the atomic number of unpaired atoms and bound molecules as a function of the single particle energy (normalized to $E_{r}$ ) and momentum $k_{x}$ (normalized to $k_{r}$ ).

tial state (Fig. 3(c)) is obtained from the measured spectrum (Fig. 3(b)). The reconstructed energy-momentum dispersion simultaneously presents the characteristics of the unpaired atoms and bound molecules in BEC side, in which the unpaired atoms show nearly quadratic dispersion, however the bound molecules does not exhibit the exactly quadratic dispersion. The bound molecules are broken into two free atoms with equal and opposite momentum in the molecule center-of-mass frame by the Raman laser. Thus there is the extended momentum distribution at the lower negative energy below the binding energy. The broader dispersion of the bound molecules is shown in Fig. 3c. In Ref. [13, 14], an inverse Abel transform is used to reconstruct the three-dimensional momentum distribution when consider the momentum distribution is isotropic. Then the dispersion spectra along the radial direction of momentum are obtained. Here, we give the dispersion in $k_{x}$ direction after integrating one direction of the absorption image. Although the dispersion spectra between our work and Ref. [13, 14] are plotted with the different forms, the dispersion spectra of the bound molecules show the similar features since they come from the same wave-function of bound molecules.

In conclusion, we experimentally study the Raman spectroscopy of ultracold Fermi gas on the BEC side near a s-wave Feshbach resonance. Since Raman process will transfer a large momentum into atoms, Raman spectroscopy presents the inherent momentum-resolved characteristics. The binding energy of molecules can directly be measured in a single running experiment by means of Raman spectroscopy. The spectral function of the ultracold Fermi gas is reconstructed, which presents the different characteristics of the unpaired atoms and bound molecules in BEC side. This experimental technology has some advantages compared to RF and Bragg technology and can be easily extended to perform spatially momentum-resolved Raman spectroscopy and to obtain the spectra function for the response of a homogeneous system in the local density approximation.

${ }^{\dagger}$ Corresponding author email: jzhang74@yahoo.com, jzhang74@sxu.edu.cn

J. Zhang would like to thank Hui Hu for helpful discussions. This research is supported by National Basic Research Program of China (Grant No. 2011CB921601), NSFC Project for Excellent Research Team (Grant No. 61121064), Doctoral Program Foundation of Ministry of Education China (Grant No. 20111401130001).

[1] S. Giorgini, L. P. Pitaevskii, and S. Stringari, Rev. Mod. Phys. 80, 1215 (2008).

[2] I. Bloch, J. Dalibard, W. Zwerger, Rev. Mod. Phys. 80, 885 (2008).

[3] C. A. Regal and D. S. Jin, Phys. Rev. Lett. 90, 230404 (2003).

[4] M. Bartenstein, A. Altmeyer, S. Riedl, R. Geursen, S. Jochim, C. Chin, J. Hecker Denschlag, R. Grimm, A. Simoni, E. Tiesinga, C. J. Williams, and P. S. Julienne, Phys. Rev. Lett. 94, 103201 (2005).

[5] C. H. Schunck, M. W. Zwierlein, C. A. Stan, S. M. F. Raupach, W. Ketterle, A. Simoni, E. Tiesinga, C. J. Williams, and P. S. Julienne, Phys. Rev. A 71, 045601 (2005).

[6] C. Chin, R. Grimm, P. Julienne and E. Tiesinga, Rev. Mod. Phys. 82, 1225 (2010).

[7] C. A. Regal, C. Ticknor, J. L. Bohn, and D. S. Jin, Nature 424, 47 (2003).

[8] S. Jochim, M. Bartenstein, A. Altmeyer, G. Hendl, S. Riedl, C. Chin, J. Hecker Denschlag, and R. Grimm, Science 302, 2101 (2003).

[9] C. H. Schunck, Y. Shin, A. Schirotzek and W. Ketterle, Nature 454, 739 (2008).

[10] M. W. Zwierlein, A. Schirotzek, C. H. Schunck, and W. Ketterle, Science 311, 492 (2006). 
[11] G. B. Partridge, W. Li, R. I. Kamar, Y. Liao, and R. G. Hulet, Science 311, 503 (2006).

[12] C. H. Schunck, Y. Shin, A. Schirotzek and W. Ketterle, Nature 454, 739 (2008).

[13] J. T. Stewart, J. P. Gaebler, and D. S. Jin, Nature 454, 744 (2008).

[14] J. P. Gaebler, J. T. Stewart, T. E. Drake, D. S. Jin, A. Perali, P. Pieri and G. C. Strinati, Nature Phys. 6, 569 (2010).

[15] Q. Chen, K. Levin, Phys. Rev. Lett. 102, 190402 (2009).

[16] Q. Chen, Y, He, C. Chien, K. Levin, Rep. Prog. Phys. 72, 122501 (2009).

[17] P. Pieri, A. Perali, G. C. Strinati, S. Riedl, M. J. Wright, A. Altmeyer, C. Kohstall, E. R. Sánchez Guajardo, J. Hecker Denschlag, and R. Grimm, Phys. Rev. A 84, 011608 (2011).

[18] M. Feld, B. Fröhlich, E. Vogt, M. Koschorreck, and M. Köhl, Nature 480, 75 (2011).

[19] A. T. Sommer, L. W. Cheuk, M. J. H. Ku, W. S. Bakr, and M. W. Zwierlein, Phys. Rev. Lett. 108, 045302 (2012).

[20] G. Veeravalli, E. Kuhnle, P. Dyke, and C. J. Vale, Phys. Rev. Lett. 101, 250403 (2008).

[21] E. D. Kuhnle, S. Hoinka, H. Hu, P. Dyke, P. Hannaford and C. J. Vale, New J. Phys. 13, 055010 (2011).

[22] G. M. Bruun and G. Baym, Phys. Rev. Lett. 93, 150403 (2004).

[23] I. Carusotto and Y. Castin, Phys. Rev. Lett. 94, 223202 (2005).

[24] L. Jiang, H. Pu, W. Zhang, and H. Y. Ling, Phys. Rev. A 80, 033606 (2009).

[25] T.-L. Dao, A. Grorges, J. Dalibard, C. Salomon, and I. Carusotto, Phys. Rev. Lett. 98, 240402 (2007).

[26] T.-L. Dao, I. Carusotto, and A. Georges, Phys. Rev. A 80, 023627 (2009).

[27] P. Wang, Z. Fu, L. Huang, and J. Zhang, Phys. Rev. A 85, 053626 (2012).

[28] D. Xiong, H. Chen, P. Wang, X. Yu, F. Gao and J. Zhang, Chin. Phys. Lett. 25, 843 (2008).

[29] D. Xiong, P. Wang, Z. Fu, and J. Zhang, Opt. Exppress. 181649 (2010).

[30] D. Xiong, P. Wang, Z. Fu, S. Chai, and J. Zhang, Chin. Opt. Lett. 8,627 (2010).

[31] P. Wang, L. Deng, E. W. Hagley, Z. Fu, S. Chai, J. Zhang, Phys. Rev. Lett. 106, 210401 (2011).

[32] P. Wang, Z. Yu, Z. Fu, J. Miao, L. Huang, S. Chai, H. Zhai, J. Zhang, arXiv:1204.1887. 